\title{
SOLUTION TO A PARABOLIC EQUATION \\ WITH INTEGRAL TYPE BOUNDARY CONDITION
}

\author{
IGNACIO BARAADAS \\ Centro de Investigación en Matemáticas, A.C. \\ Apartado Postal 402 \\ 36000 - Guanajuato Gto. México \\ SALVADOR PEREZ-ESTEVA \\ Instituto de Matemáticas, \\ Universidad Nacional Autónoma de México, \\ Ciudad Universitaria, \\ 04510, México, D.F., México.
}

(Received September 28, 1992 and in revised form January 21, 1993)

ABSTRACT In this paper we study the existence, and continuous dependence of the solution $\boldsymbol{v}=$ $\vartheta(x, t)$ on a Hölder space $H^{2+\gamma, 1+\gamma / 2}\left(\bar{Q}_{\tau}\right)\left(\bar{Q}_{\tau}=[0,1] \times[0, \tau], \quad 0<\gamma<1\right)$ of a linear parabolic equation, prescribing $\vartheta(x, 0)=f(x), \vartheta_{x}(1, \tau)=g(\tau)$ the integral type condition $\int_{0}^{b} \vartheta(x, \tau) d x=E(\tau)$.

KEY WORD AND PHRASES. Parabolic equation, integral boundary condition. 1991 AMS SUBJECT CLASSIFICATION CODE: 35K20.

\section{INTRODUCTION.}

Consider the problem of finding $\vartheta=\vartheta(x, \tau)$ such that

$$
\begin{aligned}
\vartheta_{\tau} & =\left(r(x, \tau) \vartheta_{x}\right)_{x}, & & 0<x<1, \quad 0<\tau \leq \mathcal{T}, \\
\vartheta_{x}(1, \tau) & =g(\tau), & & 0 \leq \tau \leq \mathcal{T}, \\
\vartheta(x, 0) & =f(x), & & 0 \leq x \leq 1, \\
\int_{0}^{b} \vartheta(x, \tau) d x & =E(\tau), & & 0 \leq \tau \leq \mathcal{T},
\end{aligned}
$$

with $E(0)=\int_{0}^{b} f(x) d x$, for $b$ fixed with $0<b<1$ and $r(x, \tau) \geq r_{0}>0$ on $[0,1] \times[0, \mathcal{T}]$.

In Cannon, Yanpin Lin [1] it is proved a result on existence, uniqueness and continuous dependence for this problem. In this paper we give conditions for which the solution of (1.1)-(1.4) belongs to a Hölder space and we prove that this solution depends continuously upon the data with respect to the corresponding Hölder norms. Similar problems are considered in $[2,3,5,6,8,9,10]$.

Notice that function $\vartheta$ satisfies (1.1)-(1.4) if and only if $u(x, t)=\vartheta(x, \tau)$, with $t=\int_{0}^{\tau} \frac{d \bullet}{r(b, s)}$, 
satisfies

$$
\begin{array}{rlrl}
u_{t} & =u_{x x}+\left[\left(\frac{a(x, t)-a(b, t)}{a(b, t)}\right) u_{x}\right]_{x} \\
& =\left(\frac{a(x, t)}{a(b, t)} u_{x}\right), \quad 0<x<1, \quad 0<t \leq T, \\
u_{x}(1, t) & =\tilde{g}(t), & & 0<t \leq T, \\
u(x, 0) & =f(x), & & 0 \leq x \leq 1, \\
\int_{0}^{b} u(x, t) d x & =\tilde{E}(t), & & 0 \leq t \leq T,
\end{array}
$$

where $\tilde{E}(t)=E(\tau), \tilde{g}(t)=g(\tau), a(x, t)=r(x, \tau), T=\int_{0}^{T} \frac{d s}{r(b, 0)}$, and $\tilde{E}(0)=E(0)=\int_{a}^{b} f(x) d x$.

(A) and (B) will denote problems (1.1)-(1.4) and (1.5)-(1.8), respectively. The results on existence, uniqueness and continuous dependence will be based on a standard fixed point argument for a contraction defined on a subset of an appropriate functional space. We shall follow Ladyzenskaja et al. [11] to define the spaces of Hölder continuous functions:

Let $Q_{T}=(0,1) \times(0, T), \bar{Q}_{T}=[0,1] \times[0, T]$. For $M>0, k=0,1,2$ and $0<\gamma<1, H^{k+\gamma}[0, M]$ shall denote the spaces of functions $h=h(t)$ in $[0, M]$, with $\|h\|_{M}^{(k+\gamma)}<\infty$; where

$$
\begin{gathered}
\|h\|_{M}^{(k+\gamma)}=\sum_{n=0}^{k}\left\|h^{(n)}\right\|_{M}+\left\|h^{(k)}\right\|_{M}^{(\gamma)}, \\
\|h\|_{M}=\sup _{t \in[0, M]}|h(t)|, \\
\|h\|_{M}^{(\gamma)}=|h(0)|+\sup _{t, t^{\prime} \in[0, M]} \frac{\left|h(t)-h\left(t^{\prime}\right)\right|}{\left|t-t^{\prime}\right|^{\gamma}},
\end{gathered}
$$

where $h^{(n)}$ denotes the derivative of $h$ of order $n$.

For $u: \bar{Q}_{T} \rightarrow \mathbf{R}$, let

$$
\begin{gathered}
H_{x, \gamma}^{T}(u)=\sup _{\substack{s, x^{\prime} \in[0,0] \\
t \in[0, T]}} \frac{\left|u(x, t)-u\left(x^{\prime}, t\right)\right|}{\left|x-x^{\prime}\right|^{\gamma}} \\
H_{t, \gamma}^{T}(u)=\sup _{\substack{s \in[0,0) \\
t, t^{\prime} \in[0, T]}} \frac{\left|u(x, t)-u\left(x, t^{\prime}\right)\right|}{\left|t-t^{\prime}\right|^{\gamma}} \\
\|u\|_{Q_{T}}=\sup _{(x, t) \in Q_{T}}|u(x, t)|
\end{gathered}
$$

Then $H^{\gamma, \gamma / 2}\left(\bar{Q}_{T}\right)$ and $H^{2+\gamma, 1+\gamma / 2}\left(\bar{Q}_{T}\right)$ will denote the space of all functions $u: \bar{Q}_{T} \rightarrow \mathbf{R}$ such that

$$
\|u\|_{T}^{\gamma, \gamma / 2}=\|u\|_{Q_{T}}+H_{x, \gamma}^{T}(u)+H_{t, \gamma / 2}^{T}(u)<\infty
$$


and

$$
\begin{aligned}
\|u\|_{T}^{2+\gamma, 1+\gamma / 2} & =\|u\|_{Q_{T}}+\left\|u_{x}\right\|_{Q_{T}}+\left\|u_{x x}\right\|_{Q_{T}}+\left\|u_{t}\right\|_{Q_{T}} \\
& +H_{t, \frac{\gamma+1}{2}}^{T}\left(u_{x}\right)+H_{x, \gamma}^{T}\left(u_{t}\right)+H_{x, \gamma}^{T}\left(u_{x x}\right)<\infty
\end{aligned}
$$

respectively.

$K=K(x, t)$ will denote the fundamental solution to the heat equation

$$
K(x, t)=\frac{1}{(4 \pi t)^{1 / 2}} e^{-\frac{x^{2}}{4 t}}, x \in \mathbf{R} ; t>0,
$$

and $\theta=\theta(x, t)$ shall be the Theta function

$$
\theta(x, t)=\sum_{m=-\infty}^{\infty} K(x+2 m, t),(\text { see }[4]) .
$$

\section{EXISTENCE, UNIQUENESS AND CONTINUOUS DEPENDENCE.}

DEFINITION. A function $u(x, t)$ on $\bar{Q}_{T}$ is called a solution of problem (B), if

1) $u$ and $u_{x}$ are continuous in $\bar{Q}_{T}$,

2) $u_{x x}$ is bounded in $\bar{Q}_{T}$,

3) $u$ satisfies (1.5)-(1.8).

We notice that if $u$ is such that $u_{x}$ is continuous in $\bar{Q}_{T}$ and satisfies (1.5)-(1.7), then $u$ is a solution of problem (B) if and only if

$$
a(b, t) \tilde{E}^{\prime}(t)=a(b, t) u_{x}(b, t)-a(0, t) u_{x}(0, t)
$$

or

$$
E^{\prime}(\tau)=r(b, \tau) \vartheta_{x}(b, \tau)-r(0, \tau) \vartheta_{x}(0, \tau),
$$

for $0 \leq \tau \leq \mathcal{T}, 0 \leq t \leq T$, provided $E$ is differentiable.

We shall assume the following compatibility hypothesis:

H1) $\tilde{g}(0)=f^{\prime}(1)$,

H2) $a(b, 0) \tilde{E}^{\prime}(0)=a(b, 0) f^{\prime}(b)-a(0,0) f^{\prime}(0)$, and the regularity conditions:

R1) $\tilde{E} \in H^{1+\left(\frac{1+\alpha}{2}\right)}[0, T], \tilde{g} \in H^{\frac{1+\gamma}{2}}[0, T], f \in H^{2+\gamma}$,

R2) $a, a_{x}, a_{x x} \in H^{\gamma, \gamma / 2}\left(\bar{Q}_{T}\right)$ and $H_{x, \delta}^{T}\left(a_{t}\right)<\infty$ for some $\delta>0$.

Let $V_{T}=\left\{\varphi \in H^{\left(\frac{1+x}{2}\right)}[0, T]: \varphi(0)=f^{\prime}(0)\right\}$. We define a nonlinear operator $\mathcal{F}: V_{T} \rightarrow V_{T}$ as follows: For $\varphi \in V_{T}$, let $u^{\varphi}$ be the unique solution in $H^{2+\gamma, 1+\gamma / 2}\left(\bar{Q}_{T}\right)$ of $(1.5)-(1.7)$, with $u_{x}(0, t)=$ $\varphi(t),(\mathrm{cf}[11]$, Theorem 5.3 p. 320). Then we define

$$
\mathcal{F} \varphi(t)=\frac{a(b, t)}{a(0, t)}\left(u_{x}^{\varphi}(b, t)-\tilde{E}^{\prime}(t)\right)
$$

Since $u^{\varphi} \in H^{2+\gamma, 1+\gamma / 2}\left(\bar{Q}_{T}\right)$ and (H2) holds, we have $\mathcal{F} \varphi \in V_{T}$, furthermore, if $\varphi$ is a fixed point of $\mathcal{F}$ then $u^{\varphi}$ is a solution of problem (B) and conversely. 
LEMMA 2.1. There exists $\epsilon>0$ not depending on $f, \tilde{g}, \tilde{E}$, such that if $0<T^{*}<\epsilon$ then

a) $\|\mathcal{F} \varphi-\mathcal{F} \psi\|_{T^{\bullet}} \leq \frac{1}{2}\|\varphi-\psi\|_{T^{\bullet}}, \quad \varphi, \psi \in V_{T}$

b) $\|\mathcal{F} \varphi-\mathcal{F} \psi\|_{T}^{\left(\frac{1+x}{2}\right)} \leq \frac{1}{2}\|\varphi-\psi\|_{T}^{\left(\frac{1+x}{2}\right)}, \varphi, \psi \in V_{T}$.

PROOF. Let $T^{*} \leq T, \varphi$ and $\psi$ in $V_{T^{*},} h=\varphi-\psi$ and $w=u^{\varphi}-u^{\psi}$. Then

$$
\begin{aligned}
w(x, t)= & -2 \int_{0}^{t} \theta(x, t-\tau) h(\tau) d \tau+ \\
& \int_{0}^{t} \int_{0}^{1}\{\theta(x-\xi, t-\tau)+\theta(x+\xi, t-\tau)\} F(\xi, \tau) d \xi d \tau,
\end{aligned}
$$

with $F(x, t)=\left(\frac{a(s, t)-a(b, t)}{a(b, t)} w_{x}\right)_{x}$ (cf $[4]$ p. 339).

It follows that for $t \in\left[0, T^{*}\right]$,

$$
\begin{aligned}
w_{x}(b, t)= & -2 \int_{0}^{t} \theta_{x}(b, t-\tau) h(\tau) d \tau \\
& +\int_{0}^{t} \int_{0}^{1} \theta_{x}(b+\xi, t-\tau) F(\xi, \tau) d \xi d \tau+\cdot \int_{0}^{t} \int_{0}^{1} \theta_{x}(b-\xi, t-\tau) F(\xi, \tau) d \xi d \tau \\
= & I_{1}+I_{2}+I_{3} .
\end{aligned}
$$

We clearly have

$$
\left|I_{1}\right| \leq 2\|\varphi-\psi\|_{T} \cdot \int_{0}^{T^{*}}\left|\theta_{x}(b, \tau)\right| d \tau \leq C_{1} T^{*}\|h\|_{T \cdot}
$$

Since term by term differentiation of the series in $I_{2}$ is possible, then we have

$$
\begin{aligned}
I_{2}= & \int_{0}^{t} \int_{0}^{1} \theta_{x}(b+\xi, t-\tau)\left(\frac{a(\xi, \tau)-a(b, \tau)}{a(b, \tau)} w_{\xi}(\xi, \tau)\right)_{\xi} d \xi d \tau \\
= & -\int_{0}^{t} \theta_{x}(b, t-\tau)\left(\frac{a(0, \tau)-a(b, \tau)}{a(b, \tau)}\right) w_{\xi}(0, \tau) d \tau \\
& -\int_{0}^{t} \int_{0}^{1} \theta_{x x}(b+\xi, t-\tau)\left(\frac{a(\xi, \tau)-a(b, \tau)}{a(b, \tau)}\right) w_{\xi}(\xi, \tau) d \xi d \tau .
\end{aligned}
$$

Condition (R2) implies that equation (1.5) (satisfied by $w$ ) can be differentiated (see [7, Sec. 3.5]) and then $w_{x}$ satisfies a linear parabolic equation. Thus, by the weak maximun principle it follows that

$$
\left\|w_{x}\right\|_{\bar{Q}_{T^{*}}} \leq e^{M T^{*}}\|\varphi-\psi\|_{T^{*}}=e^{M T^{*}}\|h\|_{T^{*}}, \text { where } M=\sup _{\bar{Q}_{T}}\left|\left(\frac{a(x, t)}{a(b, t)}\right)_{x=}\right|,
$$

(cf. [7, Th. 2.3.8]).

Then $\left|I_{2}\right| \leq C_{2} T^{*}\|h\|_{T^{*}}$. Finally, if we write $\theta(x, t)=K(x, t)+H(x, t)$, with $H(x, t)=$ $\sum_{\substack{m=-\infty \\ m \neq 0}}^{\infty} K(x+2 m, t)$, then

$$
\begin{aligned}
I_{3}= & \int_{0}^{t} \int_{0}^{1} H_{x}(b-\xi, t-\tau) F(\xi, \tau) d \xi d \tau \\
& +\int_{0}^{t} \int_{0}^{1} K_{x}(b-\xi, t-\tau) F(\xi, \tau) d \xi d \tau \\
= & J_{1}+J_{2} .
\end{aligned}
$$


$J_{1}$ can be estimated just as $I_{2}$, to obtain

$$
\left|J_{1}\right| \leq C_{3} T^{*}\|h\|_{T} \cdot \text { for } t \leq T .
$$

To estimate $J_{2}$ we have to take case of the singularity of $K(x, t)$ at $(0,0)$.

Since $\left|\frac{a(\xi, \tau)-a(b, \tau)}{a(b, \tau)}\right| \leq C_{4}|\xi-b|$, then integrating by parts as before, we have

$$
\begin{aligned}
\left|J_{2}\right| \leq & \int_{0}^{t}\left|K_{x}(, t-\tau)\left(\frac{a(0, \tau)-a(b, \tau)}{a(b, \tau)}\right) w_{\xi}(0, \tau)\right| d \tau \\
& +C_{5}\|h\|_{T \cdot} \int_{0}^{t} \int_{0}^{1}\left|K_{x x}(b-\xi, t-\tau)(\xi-b)\right| d \xi d \tau \\
\leq & C_{6}\left(T^{*}+T^{* 1 / 2}\right)\|h\|_{T^{*}} .
\end{aligned}
$$

Hence $\left|w_{x}(b, t)\right| \leq\left|I_{1}\right|+\left|I_{2}\right|+\left|I_{3}\right| \leq C T^{* 1 / 2}\|h\|_{T^{*}, t} \leq T^{*}$, where $C$ depends on $T, b$ and function $a(x, t)$. From this (a) follows immediately.

Now we estimate $\left\|w_{x}(b, \cdot)\right\|_{T^{*}}^{\left(\frac{2+x}{2}\right)}$ :

For $t<s$ we have

$$
\begin{aligned}
w_{x}(b, s)-w_{x}(b, t)= & -2 \int_{0}^{t} \theta_{x}(b, \tau)(h(s-\tau)-h(t-\tau)) d \tau \\
& -2 \int_{t}^{0} \theta_{x}(b, \tau) h(s-\tau) d \tau \\
& +\int_{0}^{t} \int_{0}^{1} \theta_{x}(b+\xi, \tau)(F(\xi, s-\tau)-F(\xi, t-\tau)) d \xi d \tau \\
& +\int_{t}^{s} \int_{0}^{1} \theta_{x}(b+\xi, \tau) F(\xi, s-\tau) d \xi d \tau \\
& +\int_{0}^{t} \int_{0}^{1} H_{x}(b-\xi, \tau)(F(\xi, s-\tau)-F(\xi, t-\tau)) d \xi d \tau \\
& +\int_{t}^{s} \int_{0}^{1} H_{x}(b-\xi, \tau) F(\xi, s-\tau) d \xi d \tau \\
& +\int_{0}^{t} \int_{0}^{1} K_{x}(b-\xi, \tau)(F(\xi, s-\tau)-F(\xi, t-\tau)) d \xi d \tau \\
& +\int_{t}^{e} \int_{0}^{1} K_{x}(b-\xi, \tau) F(\xi, s-\tau) d \xi d \tau \\
= & L_{1}+L_{2}+L_{3}+L_{4}+L_{3}+L_{6}+L_{7}+L_{8} .
\end{aligned}
$$

We claim that

$$
\begin{aligned}
& \left|L_{i}\right| \leq M_{i} T^{*}\|h\|_{T^{2}}^{\left(\frac{1+\tau}{2}\right)}|s-t|^{\frac{1+\gamma}{2}}, \quad i=1, \ldots, 6, \\
& \left|L_{7}\right| \leq M_{7} T^{* \delta / 2}\|h\|_{T^{\circ}}^{\left(\frac{1+\tau}{2}\right)}|s-t|^{\frac{1+\gamma}{2}}, \\
& \left|L_{8}\right| \leq M_{8} T^{* \frac{1}{2}}\|h\|_{T^{\circ}}^{\left(\frac{1+x}{2}\right)}|s-t|^{\frac{1+\gamma}{2}},
\end{aligned}
$$

where $M_{i}$ depends on $T, b$ and function $a(x, t), i=1, \ldots, 8$.

The proof of (2.4) follows as the proof of part (a). For (2.5) we let $c(x, t)=\frac{a(x, t)-a(b, t)}{a(b, t)}$, then

$$
L_{7}=-\int_{0}^{t} K_{x}(b, \tau)\left(c(0, s-\tau) w_{x}(0, s-\tau)-c(0, t-\tau) w_{x}(0, t-\tau)\right) d \tau
$$




$$
\begin{aligned}
& +\int_{0}^{t} \int_{0}^{1} K_{x x}(b-\xi, \tau) c(\xi, s-\tau)\left(w_{x}(\xi, s-\tau)-w_{x}(\xi, t-\tau)\right) d \xi d \tau \\
& +\int_{0}^{t} \int_{0}^{1} K_{x x}(b-\xi, \tau)\left(w_{x}(\xi, t-\tau)(c(\xi, s-\tau)-c(\xi, t-\tau)) d \xi d \tau\right. \\
& =J_{1}+J_{2}+J_{3} .
\end{aligned}
$$

Since $c(\xi, t)=\mathcal{O}(|\xi-b|)$, we obtain

$$
\begin{aligned}
& \left|J_{1}\right| \leq K_{1} T^{*}\|h\|_{T^{*}}^{\left(\frac{1+\gamma}{2}\right)}|t-s|^{\frac{1+\gamma}{2}} \\
& \left|J_{2}\right| \leq K_{2} T^{* 1 / 2}\|w\|_{T^{*}}^{2+\gamma, 1+\gamma / 2}|t-s|^{1+\gamma},
\end{aligned}
$$

and by (R2),

$$
J_{3}=\int_{0}^{t} \int_{0}^{1}|\xi-b|^{\delta} K_{x x}(b-\xi, \tau) w_{x}(\xi, t-\tau) \int_{t}^{i} \frac{\partial}{\partial r} \frac{c(\xi, r-\tau)}{|\xi-b|^{\delta}} d \xi d \tau
$$

Hence

$$
\left|J_{3}\right| \leq K_{3} T^{* \delta / 2}\|w\|_{T^{\bullet}}^{2+\gamma, 1+\gamma / 2}|t-s| .
$$

We obtain (2.5) from (2.7), (2.8), (2.9) and the fact that $\|w\|_{T^{\circ}}^{2+\gamma, 1+\gamma / 2} \leq M\|h\|_{T^{\circ}}^{\left(\frac{1+\gamma}{2}\right)}$, where $M$ does not depend on $T^{*}$ (see [11] Theorem 5.4, p. 322). With a similar argument we obtain (2.6), and the proof of the Lemma follows from (2.4) (2.5) and (2.6).

REMARK. Notice that Lemma 2.1(a) holds for any two functions $\varphi, \psi$ for which $u^{\varphi}, u^{\psi}$ are well defined, $u_{x}^{\varphi}, u_{x}^{\psi}$ are continuous in $\bar{Q}_{T^{*}}$ and $u_{x x}^{\varphi}, u_{x x}^{\psi}$ are bounded in $\bar{Q}_{T}$.

THEOREM 2.2. Assume that $H_{1}, H_{2}, R_{1}, R_{2}$ hold. Then there exists a unique solution $u=u(x, t)$ of Problem (B). This solution belongs to $H^{2+\gamma, 1+\gamma / 2}\left(\bar{Q}_{T}\right)$ and satisfies

$$
\|u\|_{T}^{2+\gamma, 1+\gamma / 2} \leq C(T)\left\{\|\tilde{E}\|^{1+\left(\frac{1+\gamma}{2}\right)}+\|\tilde{g}\|_{T}^{\left(\frac{1+\gamma}{2}\right)}+\|f\|_{1}^{2+\gamma}\right\} .
$$

PROOF. Let $\epsilon>0$ as in Lemma 2.1 and $T^{*}<\epsilon$, then if we define the sequence $\varphi_{1}(x)=$ $f^{\prime}(0), \varphi_{i+1}=\mathcal{F} \varphi_{i}, i=1,2 \ldots$, then Lemma 2.1 implies that the sequence of restrictions $\left\{\varphi_{i} \mid\left[0, T^{\bullet}\right]\right\}_{i \in \mathbb{N}}$ converges in $C\left[0, T^{*}\right]$ and in $H_{T^{*}}^{\left(\frac{1+x}{*}\right)}$ to a function $\varphi_{0}$.

Furthermore

$$
\begin{aligned}
& \left\|\varphi_{n}\right\|_{T^{*}}^{\left(\frac{1+\gamma}{2}\right)} \leq \sum_{i=1}^{\infty}\left\|\varphi_{i+1}-\varphi_{i}\right\|_{T^{*}}^{\left(\frac{1+\gamma}{2}\right)}+\left\|\varphi_{1}\right\|_{T^{*}}^{\left(\frac{1+\gamma}{2}\right)} \\
& \leq 2\left\|\varphi_{2}-\varphi_{1}\right\|_{T^{*}}^{\left(\frac{1+x}{2}\right)}+\left\|\varphi_{1}\right\|_{T^{*}}^{\left(\frac{1+x}{2}\right)} \\
& \leq C_{1}\left\{\|\tilde{E}\|_{T^{\circ}}^{1+\left(\frac{1+x}{2}\right)}+\|\tilde{g}\|_{T^{0}}^{\left(\frac{1+\alpha}{2}\right)}+\|f\|_{1}^{2+\gamma}\right\} \text {. }
\end{aligned}
$$

Then for $u: \bar{Q}_{T^{*}} \rightarrow \mathbf{R}$ defined by $u=u^{\varphi}$, we have

$$
\|u\|_{T^{\bullet}}^{2+\gamma, 1+\gamma / 2} \leq C_{2}\left\{\|\tilde{E}\|_{T^{*}}^{1+\left(\frac{1+\gamma}{2}\right)}+\|\tilde{g}\|_{T^{*}}^{\left(\frac{1+\gamma}{2}\right)}+\|f\|_{1}^{(2+\gamma)}\right\} .
$$

Hence $u$ is solution to the local problem. Since $C_{1}$ and $C_{2}$ depend on $T^{*}$ only, a global solution $u$ can be obtained by a standard step by step construction, and $u$ satisfies

$$
\|u\|_{T}^{2+\gamma, 1+\gamma / 2} \leq C\left\{\|\tilde{E}\|_{T}^{1+\left(\frac{1+\gamma}{2}\right)}+\|\tilde{g}\|_{T}^{\left(\frac{1+\gamma}{2}\right)}+\|f\|_{1}^{(2+\gamma)}\right\} .
$$


Finally, the remark after Lemma 2.1 implies that any solution of (B) in $\bar{Q}_{T}$ has to be $u$.

\section{REFERENCES}

[1] CANNON, J. R., YAMPIN LIN, J. and VAN DER HOEK, J. A Quasi-Linear Parabolic Equation with Nonlocal Boundary Condition. Rend di Matematica, Serie VII, Vol. 9, 1989, 239-264.

[2] CANNON, J. R. The Solution of the Heat Equation Subject to the Specification of Energy. Quart. Appl. Math. 21 (1963), 155-160.

[3] CANNON, J. R. and VAN DER HOEK, J. The Existence of and a Continuous Dependence Result for the Heat Equation subject to the Specification of Energy, Supplemento Bolletino Unione Matehamtica Italiana, Vol. 1 (1981), 253-282.

[4] CANNON, J. R. The one Dimensional Heat Equation, Encyclapedia of Mathematics and its Applications, Vol. 29, Addison-Wesley, New York, 1984.

[5] CANNON, J. R. and VAN DER HOEK, J. Diffusion subject to the Specification of Mass, J. Math. Anal. Appl. 15 (1986), No. 2, 517-529.

[6] DECKERT, K. L. and MAPLE, C. G. Solution for Diffusion with Integral Type Boundary Conditions, Proc. Jowa Acad. Sc. 70 (1963) pp. 354-361.

[7] FRIEDMAN, A. Partial Differential Equations of Parabolic Type, Prentice Hall, Inc., New York 1964.

[8] IONKIN, N. I. The Solution of a Boundary Value Problem in Heat Conduction with a nonClasical Boundary Condition. Defferential'nye Uraveija, 19 (177), pp. 294-304 (Differential Equations, 13 (1977) pp. 204-211).

[9] Stability of a Problem in Heat-Transfer Theory with a non-Classical Boundary Condition, Differential'nye Uraveija, 15 (1979), pp. 1279-1283 (Differential Equations, 15 (1980), pp. 911-914).

[10] KAMYNIN, L. I. A Boundary Value Problem in the Theory of Heat Conduction with a non-Classical Boundary Condition, Zh. Vichisl. Mat.; Mat. Fis., 4 (1964), pp. 1006-1024 (U.S.S.R. Comput. Math. and Math. Phys., 4 (1964), pp. 33-59).

[11] LADYZENSKAYA, Q. A., SOLONNIKOV, V. A. and URAL'CEVA, N. N., Linear and Quasilinear Equations of Parabolic Type, Vol. 23, Translations of Mathematical Monographs, American Mathematical Society, Providence, RI, 1968. 


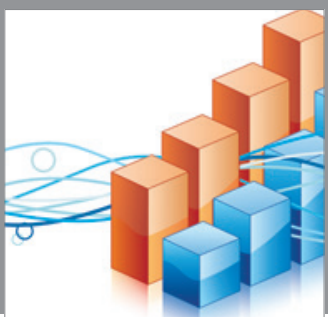

Advances in

Operations Research

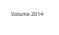

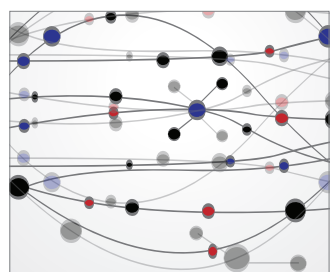

\section{The Scientific} World Journal
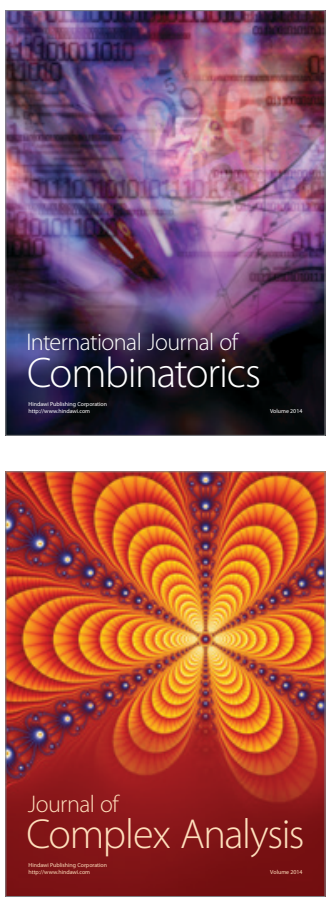

International Journal of

Mathematics and

Mathematical

Sciences
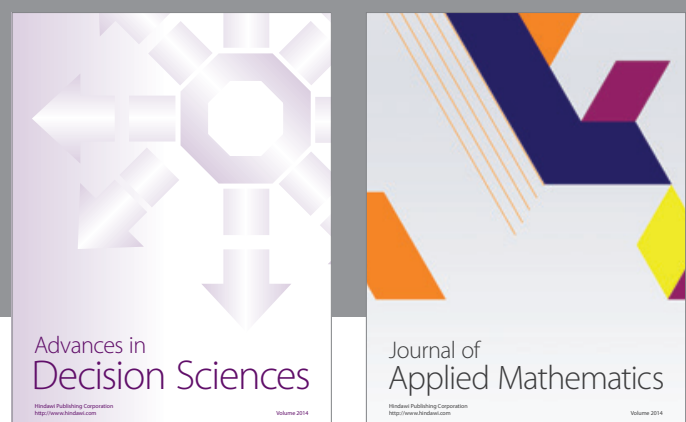

Journal of

Applied Mathematics
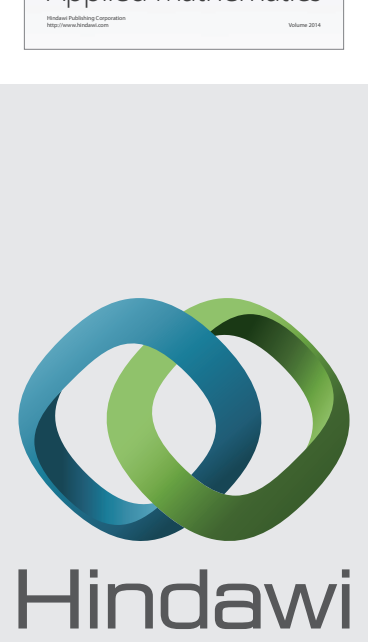

Submit your manuscripts at http://www.hindawi.com
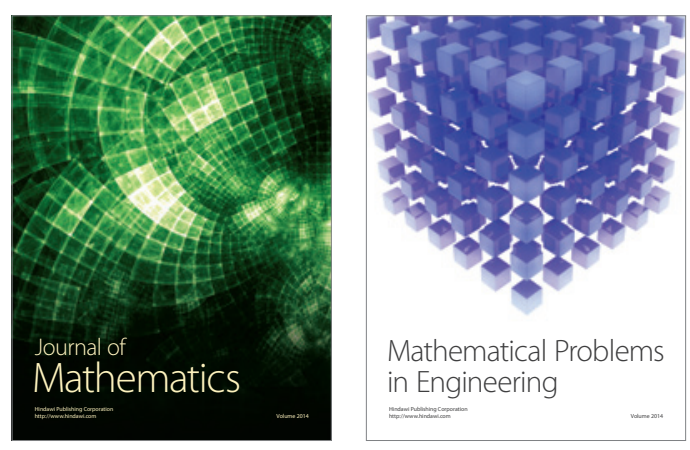

Mathematical Problems in Engineering
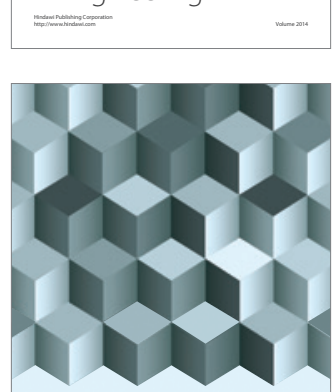

Journal of

Function Spaces
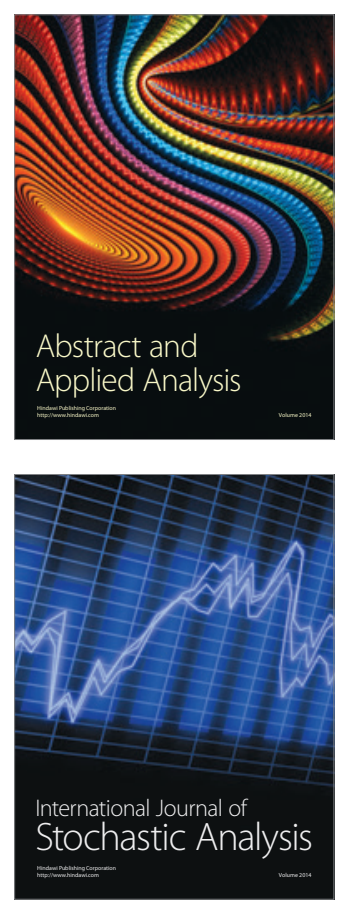

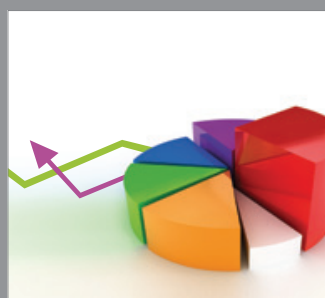

ournal of

Probability and Statistics

Promensencen
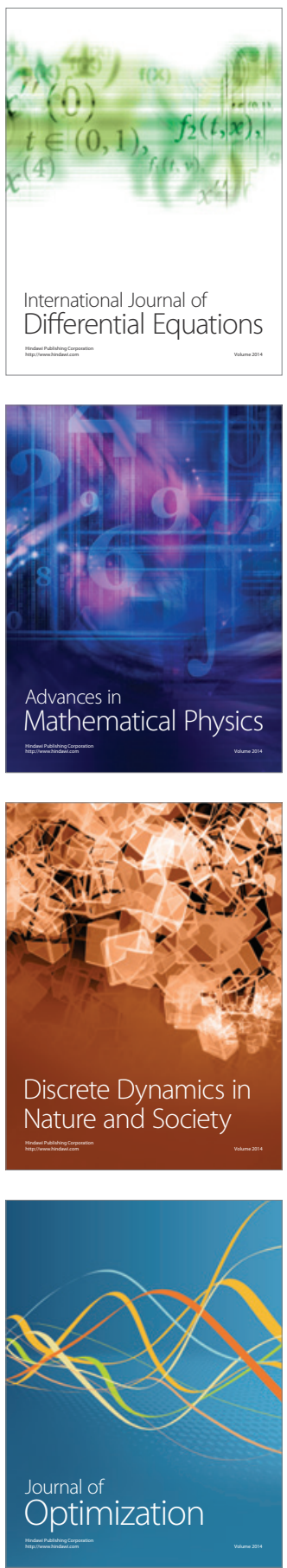